\title{
A Case-Control Study of Meat Mutagens and Colorectal Cancers in Viet Nam
}

\author{
Hang Viet Dao', Tu Thi Minh Nguyen², Hoc Hieu Tran¹, Linh Thuy Dang², Minh \\ Thi Dinh ${ }^{1}$, Ngoan Tran Le ${ }^{3,4 *}$
}

\begin{abstract}
Objective: The aim was to examine the association between heterocyclic amines 2-amino-1-methyl-6-phenylimidazo pyridine (PhIP) and the risk of colorectal cancer (CRC) in Viet Nam. Methods: We performed a case-control study for 512 colorectal cancer patients with the histopathological confirmation and 1,096 hospital controls. We collected information on lifestyle, diet, and cooking methods from participants by trained interviewers using the validated questionnaires. We used data of PhIP concentration in cooked beef analyzed by the LC/MS/MS and cooking questionnaire to estimate the daily intake of PhIP. We divided the estimated amount of PhIP (ng/person/day) into three levels of non-intake (the reference), medium, and high to estimate the Odds ratio and 95\% confidence interval (OR, 95\% CI). Results: The median intake of PhIP (ng/person/day) was $18 \mathrm{ng}$ and $102.8 \mathrm{ng}$ for medium and high PhIP intake, respectively. There was a significant association between PhIP intake and the risk of colorectal cancer. The adjusted OR $(95 \% \mathrm{C})$, high intake vs. non-intake, were $4.89(3.03,7.89)$, $p \_$trend $<0.01$ for all participants, for men OR $(95 \% \mathrm{C}): 5.27(2.83,9.81)$, $p \_$trend $<0.01$, for women OR $(95 \% \mathrm{C}): 4.58(2.10,10.01)$, $p \_$trend $<0.01$. The significant positive association was also observed for the sub-sites of the colon (proximal and distant) and rectum cancers. Conclusions: We observed the positive association of PhIP contributed from cooked-beef and the development of CRC. Cooking methods related to the occurrence of PhIP and other types of heterocyclic amines in cooked-beef and other meats would be significant investigations.
\end{abstract}

Keywords: Colorectal cancer- meat mutagens- cooking method- beef intake

Asian Pac J Cancer Prev, 21 (8), 2217-2223

\section{Introduction}

A rapid significant increase of both colorectal cancer (CRC) mortality and meat intake has been seen at populations in Viet Nam from 1981 to date. The age-standardized mortality rate per 100,000 (Segi-standard world population) in the Nghe An province continuously increased from 2.65 (in 2005-2006) to 2.80 (2007-2008), 3.30 (2009-2010), 4.24 (2011-2012), and 4.36 (2013-2014) (Thuong, 2018). Meat consumption per person per day has steadily increased from $11.1 \mathrm{~g}(1981-1985)$ to $24.4 \mathrm{~g}$ (1987-1989), 51.0g (2000), and 84.0g (2010); pork and beef are the main red meat intake in Viet Nam (Ministry of Health and National Institute of Nutrition, 2010). While the consumption of red meat is probably carcinogenic to humans and the target organs were colorectal, pancreases, and prostate (Group 2A) (IARC, 2018), the association of meat intake and the occurrence of colorectal cancer in the country and Asia region is still limited. The underlying etiology of red meat induced cancer might be dietary chemical carcinogens generated in cooked meat at high cooking temperatures. There were over 20 types of heterocyclic amines contaminated in our cooked meat. Major HCAs found in human diet include 2-amino3,8-dimethylimidazo quinoxaline (MeIQx), 2-amino1-methyl-6-phenylimidazo pyridine (PhIP), 2-amino3,4,8-trimethylimidazo (DiMeIQx). Animal studies have provided sufficient evidence for the carcinogenic potential (Group 2B-IARC) of MeIQx, DiMeIQx and PhIP (IARC, 1993; NTP, 2014; Sugimura, 1997).

Heterocyclic amines 2-amino-1-methyl-6phenylimidazo pyridine (PhIP) has detected in steak (1.9 to $30 \mathrm{ng} / \mathrm{g}$ ) but was formed only in very well done fried or grilled hamburger (Sinha et al., 1998). Animal studies have provided sufficient evidence for their carcinogenic potential (Group 2B, International Agency for Research on Cancer, IARC) of PhIP (IARC, 1993; NTP, 2014).

The aim was to examine the association between heterocyclic amines 2-amino-1-methyl-6-phenylimidazo pyridine and the risk of colorectal cancer in the North

${ }^{1}$ Hanoi Medical University, Hanoi, Viet Nam. ${ }^{2}$ School of Biotechnology and Food Technology, Hanoi University of Science and Technology, Hanoi, Viet Nam. ${ }^{3}$ Institute of Research and Development, Duy Tan University, Da Nang 550000, Viet Nam. ${ }^{4}$ Department of Public Health, School of Medicine, International University of Health and Welfare, Japan. *For Correspondence: lengoan@iuhw.ac.jp 
Viet Nam.

\section{Materials and Methods}

\section{Study population}

A case-control study performed for 512 colorectal cancers and 1096 hospital controls. The study participants recruited from the in-patient departments of three University Hospitals in the Hanoi city from Dec. 2017 to Dec. 2018.

\section{Case ascertainment}

Among a total of 3,928 recruited incidence patients, we identified 512 cases diagnosed colorectal cancer by histopathological confirmation, Figure 1. Colorectal cancers included 312 colon cancer cases and 200 rectum cancer cases. Among colon cancer, it was 102 proximal colon, 93 distal colon, and another 117 cases. Among total colorectal cancer, men were 292 , and women were 220 cases.

\section{Control recruitment}

Controls were 1096 non-cancer patients: Kidney donation (18), palm-sweating (8), gall bladder stones (168), benign prostatic hyperplasia (71), hemorrhoids (85), and herniation (74), Kidney stone (449), colorectal polyp (20), other non-cancer morbidities (203). They admitted to the same in-patient departments for weekly surgery. They were cancer-free on the recruited time and in their lifetime health history.

\section{Exclusion}

We excluded both cancer cases and controls in case they were unable to communicate due to advanced disease stages; patients having substantial changes in their diet due to metabolic disorders, and diabetes; and patients refused to participate in the study.

\section{Assessment of diet and cooking methods}

We used the validated semi-quantitative food frequency questionnaire to collect the dietary history of participants in the past year, before the onset of cancer or non-cancer diseases. The SQFFQ has good characteristics of feasibility, practical, and reliability in the general population. The SQFFQ included 97 foods/recipes of 12 groups of foods that included cereals (9 items), peanuts and beans (7) vegetables (24), fruits (10), fat and oil (3), red and white meats (22), fishes (10), eggs (4), milk and its products (3), snacks (2), salt and fish sauce (2), and alcohol or vodka (1). We classified the food frequencies of intake into seven categories: never or seldom, 6-11 times/year, 1-3 times/month, 1-2 times/week, 3-4 times/ week, 5-6 times/week, and 1-3 times/day (Le et al., 2018).

For tobacco smoking, we classified all participants into three categories: never smokers, ex-smokers, and current smokers in their lifetime. Never smokers were those who never smoked completely only one cigarette or waterpipe tobacco (WPT) smoking in their lifetime. For smokers, the information on types of tobacco (cigarette, WPT, or both types), the average number of cigarette and WPT per day during their age of 15-20, 21-25, 26-30,
31-40, 41-50, 51-60, 61-70, and 71+ (if applicable), the duration of smoking (current smokers), and the duration of quit smoking (current and ex-smokers) were obtained. Finally, we divided participants into two groups of lifestyle never-smokers and ever smokers for adjustment in the multivariable-adjusted model (Le et al., 2018).

For cooking methods, a questionnaire inquired about the frequency of intake (i.e. never or less than six times/year, 6-11/year; 1-3/month, 1-2/week, 3-4/week; 5-6/week, and 1-3/day). Also, doneness levels included four categories (i.e. lightly browned; medium browned; well browned, and blackened/charred) of cooked beef (i.e. pan-fried hamburger, pan-fried steak, roast beef, and barbecued steak). We have checked the accuracy of obtained data using this cooking questionnaire by the measurement of observer agreement for categorical data as a generalized kappa-type statistic, and estimation of sensitivity and specificity. We evaluated the repetition of cooking methods of beef in a sample of 292 participants. Each participant completed twice the cooking questionnaire one month apart during the intervening month. Participants consumed at least one of five types of cooking pan-fried hamburger; pan-fried steak, roast beef, and barbecued steak were counted as the eating group, code as 1 , the other code as 2 for both the first and second completed the cooking questionnaire. The estimated Kappa (0.55) was good and the agreement as high as $86 \%$. The estimated sensitivity $(70.6 \%)$ and specificity $(89.2 \%)$ was also very good (non-published data). Therefore, data on cooking methods of beef was reliable to estimate the amount of PhIP intake per day per person in the present study.

\section{Assessment of HCA intake}

The experience investigators examined PhIP concentration in cooked beef in the laboratories of the School of Biotechnology and Food Technology, Hanoi University of Science and Technology, and the Center for Research and Technology Transfer, Vietnam Academy of Science and Technology (Linh, 2019). They performed LC/MS/MS method to analyze PhIP and MeIQx in 14 fried and grilled food samples, represented for the regular meals of participants from three communities represented three provinces in the Red Delta River in the North Viet Nam. The estimated concentration $(\mathrm{C}, \mathrm{ng} / \mathrm{g}$ ) of PhIP was 77.2 $\mathrm{ng} / \mathrm{g}$ in the part of blackened/charred barbecued steak and $10.4 \mathrm{ng} / \mathrm{g}$ in the part of blackened/charred pan-fried steak, pan-fried hamburger, roast beef. We assumed that the $\mathrm{PhIP}$ concentration by doneness levels will be balanced (B, \%) to be $100 \%$ in the part of blackened/charred, then it was $75 \%$ in the well browned, $50 \%$ in the medium browned, and $25 \%$ in the lightly browned of cooked-beef.

The average amount (A, gram $(\mathrm{g}))$ of intake of cooked beef recipes was $33.3 \mathrm{~g}, 170.0 \mathrm{~g}, 136.5 \mathrm{~g}$, and $66.7 \mathrm{~g}$ for the pan-fried hamburger, pan-fried steak, roast beef, and barbecued steak, respectively (Linh, 2019). Based on the obtained information provided by participants of the frequency of intake (i.e. never or less than six times/year, 6-11/year; 1-3/month, 1-2/week, 3-4/week; 5-6/week, and 1-3/day), we counted a total frequency of intake (F) of beef serving times per year. The estimated amount of PhIP from the beef of intake calculated by the following 
formulation:

(A: The average amount $(\mathrm{A}, \mathrm{g})$ of intake of each cooked beef recipe; $\mathrm{B}$ : the balanced PhIP concentration by doneness levels (B, \%); C: The estimated concentration $(\mathrm{C}, \mathrm{ng} / \mathrm{g})$ of PhIP intake; F: Total frequency of intake (F) of beef serving times per year).

\section{Data handle and statistical analyses}

Body Mass Index (BMI) was calculated as (BMI= weight $(\mathrm{kg}) /$ height $\left((\mathrm{m})^{2}\right)$ for adjustment. We divided the exposure to PhIP into three levels of non-intake, medium, and high. The non-beefeater participants were the reference, crude, and adjusted Odds ratio and $95 \%$ confidence interval $(\mathrm{OR}, 95 \% \mathrm{CI})$ were estimated. Model 1: Adjusted for age groups (0-29, 30-39, $40-49,50-59,60-69, \geq 70$ ages) for men and women; and age groups and sex for a total. Model 2: Multivariable adjusted for age groups (0-29, 30-39, 40-49, 50-59, 60-69, $\geq 70$ ages) for men and women; and age groups and sex for total; BMI (18.5 to $<23,23$ to $<25, \geq 25,<18.5 \mathrm{~kg}$ / $\mathrm{m}^{2}$ ); education level (Primary school or under, secondary school, high school, higher high school, unknown); Frequency of intake of vegetables (Water spinach, mustard greens, sauropus, Malabar nightshade, cabbage); and lifetime smoking (cigarette, WPT, or both) (yes/no).

\section{Ethics consideration}

The ethic certificate of approval by the Hanoi Medical University IRB for the present study was issued on Dec. 25th, 2018 and by the International University of Health and Welfare IRB, Japan on May $17^{\text {th }}, 2019$. We obtained written informed consent from all participants of the present study.

\section{Results}

There was $30.84 \%$ of 642 controls in men and $99.34 \%$ of 454 in women to be never lifetime smokers. Among CRC cancer, there was $71.23 \%$ of 292 men and $1.82 \%$ of 220 women categorized as ever lifetime smokers. The proportion of overweight or obese in the controls was
$13.40 \%$ in men and 11.45 in women. The occurrence of CRC before the age of 30 was $1.37 \%$ in men (4 patients) and $1.36 \%$ in women (3 patients). The average age was 59.40 (25-99) in CRC patients and 54.74 (17-92) in the controls (data not shown).

Among 1,608 participants, number beefeaters of cooked-beef (i.e. pan-fried hamburger, pan-fried steak, roast beef, and barbecued steak) were 218 (12.5\%). When men and women combined, the median intake of PhIP from beef per person per day was $18 \mathrm{ng}$ and 102ng for the medium and high amount of intake per day, respectively.

Overall the multivariable adjusted risk significantly increased due to a high intake of PhIP from beef: OR (95\%CI): $4.89(3.03,7.89)$, p_trend $<0.01$ for total participants, OR $(95 \% \mathrm{CI}): 5.27(2.83,9.81)$, p_trend $<0.01$ for men, and OR $(95 \% \mathrm{CI}): 4.58(2.10,10.01), p \_$trend $<0.01$ for women. A similar observation was seen for colon OR (95\% CI): 5.68 (3.31, 9.76), p_trend $<0.01$ for total colon cancer, OR (95\%CI): $6.67(3.35,13.3), p \_$trend $<0.01$ for men, and OR $(95 \% \mathrm{CI}): 4.53(1.79,11.5), p_{-}$trend $<0.01$ for women. For rectum, OR $(95 \% \mathrm{CI}): 4.24(2.29,7.84)$, p_trend $<0.01$ for total rectum cancer, OR $(95 \% \mathrm{CI}): 3.49$ (1.48, 8.23), $p$ trend $<0.01$ for men, and OR $(95 \% \mathrm{CI}): 5.50$ $(2.10,14.39), p_{-}$trend $<0.01$ for women, Table 1.

By subsides, the significantly increased risk was observed for proximal colon, OR $(95 \% \mathrm{CI}): 8.05$ (3.86, $16.77)$, p_trend $<0.01$ for total proximal colon, OR (95\%CI): 6.91 (2.65, 18.07), p_trend $<0.01$ for men, and OR (95\%CI): $12.15(3.73,39.57), p$ trend $<0.01$ for women. For distal colon, OR (95\%CI): $1.69(0.49,5.86)$, p_trend $<0.01$ for total distal colon, OR $(95 \% \mathrm{CI}): 2.82$ $(0.75,10.52), p_{\text {_trend }}=0.05$, for men, Table 2 .

By smoking status, the elevated risk was seen for the never-lifestyle smokers, OR $(95 \% \mathrm{CI}): 4.78$ (2.55, $8.95)$, p_trend $<0.01$, men and women combined, OR (95\% CI): $5.07(1.76,14.61), p$ trend $<0.01$ for men, and OR $(95 \% \mathrm{CI}): 4.40(1.99,9.70), p_{-}$trend $<0.01$ for women. For ever-lifestyle smokers, OR $(95 \% \mathrm{CI}): 5.58$ $(2.55,12.20), p \_$trend $<0.01$, men and women combined, OR (95\%CI): $5.37(2.44,11.84)$, $p_{-}$trend $<0.01$ for men. Data was not available for women due to a small number

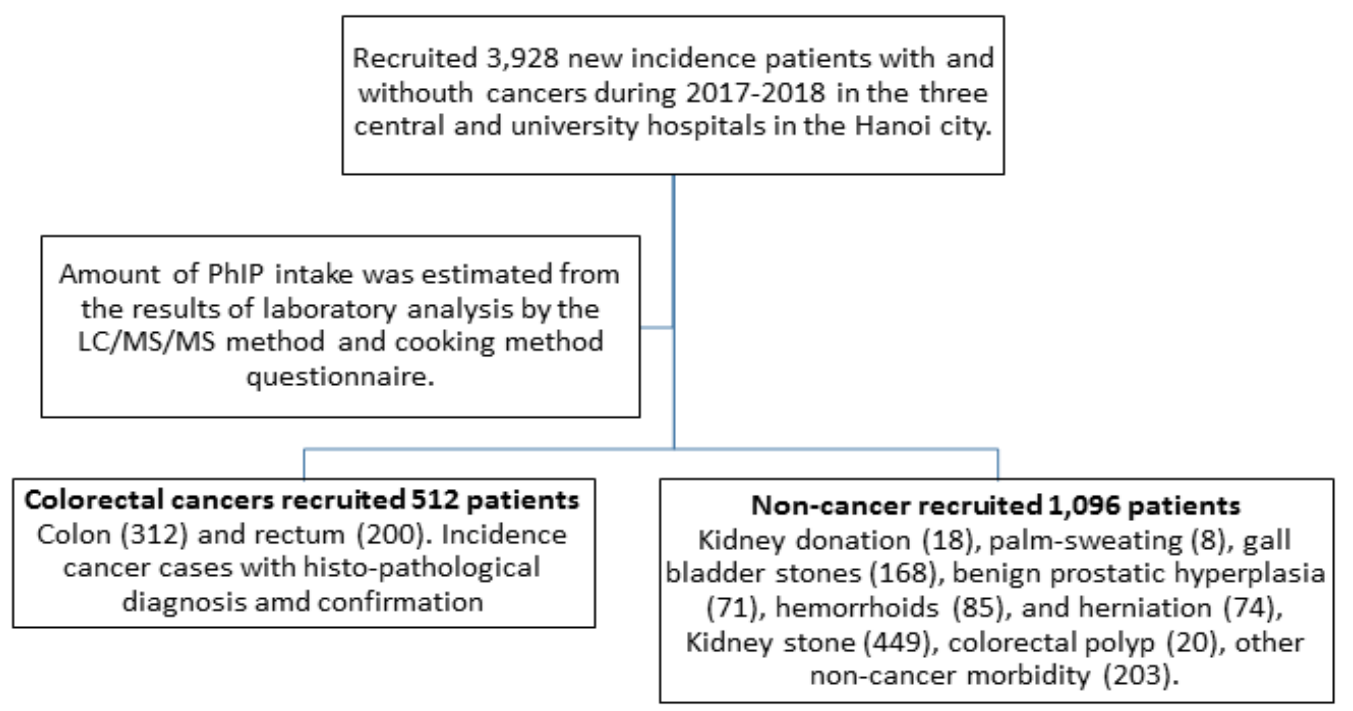

Figure 1. Flow Chart of the Recruited Study Participants 
Table 1. PhIP from Beef Intake and Risk of Colorectal Cancers

\begin{tabular}{|c|c|c|c|c|c|c|c|c|}
\hline & Control & Cancer & Total & $\begin{array}{l}\text { PhIP intake } \\
\text { (Median) }\end{array}$ & $\begin{array}{l}\text { Adjusted }{ }^{\mathrm{b}} \text { OR } \\
(95 \% \mathrm{CI})\end{array}$ & $P_{-}$trend & $\begin{array}{c}\text { Multivariable } \\
\text { adjusted }{ }^{\circ} \mathrm{OR}(95 \% \mathrm{CI})\end{array}$ & $P$ trend \\
\hline \multicolumn{9}{|c|}{$\mathrm{CRC}^{\mathrm{a}}$} \\
\hline \multirow[t]{3}{*}{ Men and women } & 1,015 & 402 & 1,417 & Non-intake & 1.00 (reference) & & 1.00 (reference) & \\
\hline & 50 & 57 & 107 & 18 & $3.27(2.17,4.93)$ & & $3.06(2.00,4.66)$ & \\
\hline & 31 & 53 & 84 & 102.8 & $4.86(3.03,7.80)$ & $<0.01$ & $4.89(3.03,7.89)$ & $<0.01$ \\
\hline Total & 1,096 & 512 & 1,608 & & & & & \\
\hline \multirow[t]{3}{*}{ Men } & 593 & 231 & 824 & Non-intake & 1.00 (reference) & & 1.00 (reference) & \\
\hline & 31 & 29 & 60 & 18 & $2.66(1.56,4.56)$ & & $2.77(1.57,4.88)$ & \\
\hline & 18 & 32 & 50 & 87 & $4.96(2.69,9.11)$ & $<0.01$ & $5.27(2.83,9.81)$ & $<0.01$ \\
\hline Total & 642 & 292 & 934 & & & & & \\
\hline \multirow[t]{3}{*}{ Women } & 422 & 171 & 593 & Non-intake & 1.00 (reference) & & 1.00 (reference) & \\
\hline & 19 & 28 & 47 & 18 & $4.30(2.25,8.21)$ & & $3.70(1.91,7.15)$ & \\
\hline & 13 & 21 & 34 & 112.5 & $4.70(2.21,9.98)$ & $<0.01$ & $4.58(2.10,10.01)$ & $<0.01$ \\
\hline Total & 454 & 220 & 674 & & & & & \\
\hline \multicolumn{9}{|c|}{ Colon } \\
\hline \multirow[t]{3}{*}{ Men and women } & 1,015 & 241 & 1,256 & Non-intake & 1.00 (reference) & & 1.00 (reference) & \\
\hline & 50 & 37 & 87 & 18 & $3.59(2.25,5.72)$ & & $3.28(2.02,5.31)$ & \\
\hline & 31 & 34 & 65 & 97.5 & $5.65(3.31,9.63)$ & $<0.01$ & $5.68(3.31,9.76)$ & $<0.01$ \\
\hline Total & 1,096 & 312 & 1,408 & & & & & \\
\hline \multirow[t]{3}{*}{ Men } & 593 & 139 & 732 & Non-intake & 1.00 (reference) & & 1.00 (reference) & \\
\hline & 31 & 19 & 50 & 18 & $2.98(1.61,5.52)$ & & $2.86(1.48,5.52)$ & \\
\hline & 18 & 23 & 41 & 76.5 & $6.47(3.31,12.67)$ & $<0.01$ & $6.67(3.35,13.3)$ & $<0.01$ \\
\hline Total & 642 & 181 & 823 & & & & & \\
\hline \multirow[t]{3}{*}{ Women } & 422 & 102 & 524 & Non-intake & 1.00 (reference) & & 1.00 (reference) & \\
\hline & 19 & 18 & 37 & 18 & $4.54(2.19,9.42)$ & & $4.48(2.11,9.5)$ & \\
\hline & 13 & 11 & 24 & 117 & $4.37(1.82,10.51)$ & $<0.01$ & $4.53(1.79,11.5)$ & $<0.01$ \\
\hline Total & 454 & 131 & 585 & & & & & \\
\hline \multicolumn{9}{|c|}{ Rectum } \\
\hline \multirow[t]{3}{*}{ Men and women } & 1,015 & 161 & 1,176 & Non-intake & 1.00 (reference) & & 1.00 (reference) & \\
\hline & 50 & 20 & 70 & 18 & $2.82(1.62,4.91)$ & & $2.73(1.54,4.82)$ & \\
\hline & 31 & 19 & 50 & 117 & $4.16(2.27,7.63)$ & $<0.01$ & $4.24(2.29,7.84)$ & $<0.01$ \\
\hline Total & 1,096 & 200 & 1,296 & & & & & \\
\hline \multirow[t]{3}{*}{ Men } & 593 & 92 & 685 & Non-intake & 1.00 (reference) & & 1.00 (reference) & \\
\hline & 31 & 10 & 41 & 18 & $2.22(1.04,4.70)$ & & $2.61(1.19,5.74)$ & \\
\hline & 18 & 9 & 27 & 117 & $3.38(1.46,7.79)$ & $<0.01$ & $3.49(1.48,8.23)$ & $<0.01$ \\
\hline Total & 642 & 111 & 753 & & & & & \\
\hline \multirow[t]{3}{*}{ Women } & 422 & 69 & 491 & Non-intake & 1.00 (reference) & & 1.00 (reference) & \\
\hline & 19 & 10 & 29 & 18 & $3.84(1.66,8.90)$ & & $3.24(1.35,7.77)$ & \\
\hline & 13 & 10 & 23 & 117 & $5.35(2.17,13.18)$ & $<0.01$ & $5.50(2.10,14.39)$ & $<0.01$ \\
\hline Total & 454 & 89 & 543 & & & & & \\
\hline
\end{tabular}

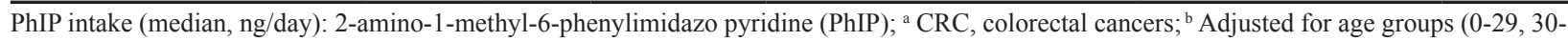
$39,40-49,50-59,60-69, \geq 70$ ages) for men and women; and age groups and sex for the total; c Multivariable adjusted for age groups $(0-29,30-39$, $40-49,50-59,60-69, \geq 70$ ages) for men and women; and age groups and sex for total; BMI ( 18.5 to $<23,23$ to $\left.<25, \geq 25,<18.5 \mathrm{~kg} / \mathrm{m}^{2}\right)$; education level (Primary school or under, secondary school, high school, higher high school, unknown); Frequency of intake of vegetables (Water spinach, mustard greens, sauropus, Malabar nightshade, cabbage); and lifetime smoking (yes/no)

of women smokers, (data not shown).

\section{Discussions}

An unhealthy diet continues to be a major public health issue worldwide, especially in low- and middle-income countries, including Viet Nam. Also, the underlying etiology of an unhealthy diet induces cancer and non-communicable diseases remain unclear to date. The present case-control study shows that PhIP 
Table 2. PhIP from Beef Intake and Risk of Sub-Sites of Proximal and Distal Colon Cancer

\begin{tabular}{|c|c|c|c|c|c|c|c|c|}
\hline & Control & Cancer & Total & $\begin{array}{l}\text { PhIP intake } \\
\text { (Median) }\end{array}$ & $\begin{array}{l}\text { Adjusted a } \\
\text { OR }(95 \% \mathrm{CI})\end{array}$ & $P_{\text {_trend }}$ & $\begin{array}{l}\text { Multivariable adjusted } \\
\text { OR }(95 \% \mathrm{CI})\end{array}$ & $P_{\text {_trend }}$ \\
\hline \multicolumn{9}{|c|}{ Proximal colon } \\
\hline \multirow[t]{3}{*}{ Men and women } & 1,015 & 75 & 1,090 & Non-intake & 1.00 (reference) & & 1.00 (reference) & \\
\hline & 50 & 13 & 63 & 18 & $4.10(2.08,8.08)$ & & $3.91(1.95,7.83)$ & \\
\hline & 31 & 14 & 45 & 97.5 & $8.56(4.17,17.55)$ & $<0.01$ & $8.05(3.86,16.77)$ & $<0.01$ \\
\hline Total & 1,096 & 102 & 1,198 & & & & & \\
\hline \multirow[t]{3}{*}{ Men } & 593 & 45 & 638 & Non-intake & 1.00 (reference) & & 1.00 (reference) & \\
\hline & 31 & 8 & 39 & 18 & $3.93(1.67,9.23)$ & & $4.05(1.66,9.89)$ & \\
\hline & 18 & 8 & 26 & 87 & $7.81(3.07,19.9)$ & $<0.01$ & $6.91(2.65,18.07)$ & $<0.01$ \\
\hline Total & 642 & 61 & 703 & & & & & \\
\hline \multirow[t]{3}{*}{ Women } & 422 & 30 & 452 & Non-intake & 1.00 (reference) & & 1.00 (reference) & \\
\hline & 19 & 5 & 24 & 18 & $4.24(1.39,12.95)$ & & $4.33(1.33,14.03)$ & \\
\hline & 13 & 6 & 19 & 117 & $9.97(3.24,30.64)$ & $<0.01$ & $12.15(3.73,39.57)$ & $<0.01$ \\
\hline Total & 454 & 41 & 495 & & & & & \\
\hline \multicolumn{9}{|c|}{ Distal colon } \\
\hline \multirow[t]{3}{*}{ Men and women } & 1,015 & 77 & 1,092 & Non-intake & 1.00 (reference) & & 1.00 (reference) & \\
\hline & 50 & 13 & 63 & 18 & $4.09(2.09,8.02)$ & & $3.34(1.66,6.73)$ & \\
\hline & 31 & 3 & 34 & 117 & $1.57(0.46,5.34)$ & $<0.01$ & $1.69(0.49,5.86)$ & 0.01 \\
\hline Total & 1,096 & 93 & 1,189 & & & & & \\
\hline \multirow[t]{3}{*}{ Men } & 593 & 38 & 631 & Non-intake & 1.00 (reference) & & 1.00 (reference) & \\
\hline & 31 & 6 & 37 & 18 & $3.52(1.36,9.13)$ & & $2.28(0.77,6.76)$ & \\
\hline & 18 & 3 & 21 & 117 & $3.07(0.85,11.15)$ & 0.01 & $2.82(0.75,10.52)$ & 0.05 \\
\hline Total & 642 & 47 & 689 & & & & & \\
\hline \multirow[t]{2}{*}{ Women } & 422 & 39 & 461 & Non-intake & 1.00 (reference) & & 1.00 (reference) & \\
\hline & 32 & 7 & 39 & 25.5 & $2.87(1.15,7.12)$ & 0.02 & $3.16(1.22,8.17)$ & 0.02 \\
\hline Total & 454 & 46 & 500 & & & & & \\
\hline
\end{tabular}

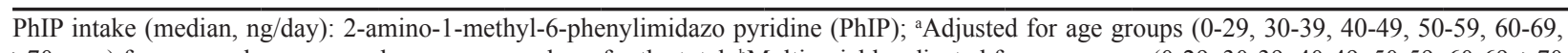

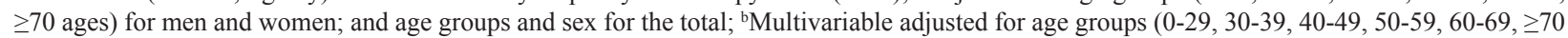
ages) for men and women; and age groups and sex for total; BMI (18.5 to $<23,23$ to $<25, \geq 25,<18.5 \mathrm{~kg} / \mathrm{m} 2$ ); education level (Primary school or under, secondary school, high school, higher high school, unknown); Frequency of intake of vegetables (Water spinach, mustard greens, sauropus, Malabar nightshade, cabbage); and lifetime smoking (yes/no)

from beef-related to the significantly increased risk of CRC in the beefeaters when compared to the reference group of non-beefeaters. This is the most comprehensive and up-to-date observational study in Viet Nam and the Asia region. We observed a strong positive association between intakes of PhIP from beef and the development of CRC in total and their sub-site of the colon (included proximal and distal colon) and rectum cancer in both men and women. The role of $\mathrm{PhIP}$ from beef-related to CRC cancer appears to be independent with tobacco smoking when a strong positive association between intakes of PhIP from beef and the development of CRC remains statistically significant in the sub-group of never lifetime smokers in both men and women. Our findings support to the hypotheses that the group of heterocyclic amines occurred in cooked meat, especially in cooked beef (i.e. pan-fried hamburger, panfried steak, roast beef, and barbecued steak) is possibly carcinogenic to humans (Group 2B) (IARC, 1993; NTP, 2014; Sugimura, 1997). Our study demonstrated that there is a significantly increased risk of CRC in both men and women, both proximal and distal colon in our study population. Innovative strategies in promoting a healthy diet to reduce consuming red meat included beef will likely be needed to prevent the occurrence of CRC and its sub-sites.

The National Toxicology Program. Department of Health and Human Services concluded: "PhIP is reasonably anticipated to be a human carcinogen based on sufficient evidence of carcinogenicity from studies in experimental animals and supporting nontoxicity data" (IARC, 1993; NTP, 2014). Heterocyclic amines included PhIP are generated when meats are cooked at high cooking temperatures resulted from the reaction between amino acids, sugars, and creatinine (Hodge, 1953; Maillard, 1912). Over 20 types of heterocyclic amines have been identified in cooked meats, and concentrations appear to increase with higher temperatures and longer duration of cooking (Sugimura, 1997; Turesky et al., 2007).

The strengths of the present study include a large sample size of $512 \mathrm{CRC}$ cases and 1,096 non-cancer controls. We used local analyzed data of PhIP concentration in cooked beef by the LC/MS/MS method that will reflect a real current intake of $\mathrm{PhIP}$ in our study population. Using the validated SQFFQ, demographic, and lifestyle cooking method questionnaires, we obtained data of food frequencies of intake of cooked beef and other foods/ 
recipes with reasonable completeness and reliable quality to estimate the amount of PhIP intake by participants. With these advanced approaches, we explored the positive association between heterocyclic amines of PhIP from beef and risk of CRC for the entire study population, in both men and women, for sub-types of the proximal and distal colon, and both never- and ever-lifetime tobacco smokers. Our findings are consistent with the previous results confirmed the positive association between heterocyclic amines intake and the risk of CRC or its sub-sites(Butler et al., 2003; Helmus et al., 2013; Joshi et al., 2015; Le Marchand et al., 2002; Miller et al., 2013; Nowell et al., 2002). The present findings were consistent with the previous study on the association between cooking method and cancer risk in the North Viet Nam. A high intake of roasted meats including beef significantly increased the risk of stomach and colorectal cancers (Ngoan et al., 2009).

A few studies have examined the relationship between heterocyclic amines included PhIP and risk of CRC, and the results are inconsistent. Some U.S. based case-control studies on HCAs and CRC observed a positive association between HCA intake and CRC or colon cancer (Butler et al., 2003; Helmus et al., 2013; Joshi et al., 2015; Le Marchand et al., 2002; Miller et al., 2013; Nowell et al., 2002). A null association between HCA intake and CRC or colon cancer was observed in the other studies (Augustsson et al., 1999; Gilsing et al., 2012; Kobayashi et al., 2009). Two large prospective cohort studies in the U.S. have previously reported the association between HCAs and the risk of CRC. However, the positive association between HCAs and the risk of CRC was seen on the NIHAARP study (Cross et al., 2010) but not in the Multiethnic Cohort study (Ollberding et al., 2012). Differences in exposure assessment using cooking questionnaires and the use of the different HCA database may at least partly explain those differential results.

Our study has some limitations. This case-control study recalled exposure data and information that is a limitation of methodological approaches. Data on PhIP intake is just available for cooked-beef, while PhIP concentration identified in many other cooked types of meat, chicken, fishes (IARC, 1993; NTP, 2014; Sugimura, 1997). In spite of these limitations, the present results warrant further investigation for heterocyclic amines, meat-derived mutagenicity (MDM), 2-amino-3,8dimethylimidazo(4,5-j)quinoxaline (MeIQx), 2-amino-1methyl-6-phenylimidazo(4,5-b)pyridine (PhIP), 2-amino3,4,8-trimethylimidazo(4,5-f)quinoxaline (DiMeIQx). For distal colon cancer, in men, only 58 participants were included in the final analytical model, and the positive association was at the borderline of statistical analysis with $p \_$trend $=0.05$, due to the relatively small number of cancer for this sub-site.

In conclusion, we observed the positive association of PhIP from beef intake and the development of CRC. Cooking methods related to the PhIP occurrence in beef recipes would be a significant investigation for our healthy diet against CRC in particular and non-communicable diseases in general.

\section{Acknowledgments}

We used the study Grant Agreement No.: 18/FIRST/1a/ HMU, Under the Project: "Fostering Innovation through Research, Science and Technology".

\section{Conflict of interest}

There are no conflicts to disclose.

\section{References}

Augustsson K, Skog K, Jagerstad M, Dickman PW, Steineck G (1999). Dietary heterocyclic amines and cancer of the colon, rectum, bladder, and kidney: a population-based study. Lancet, 353, 703-7.

Butler LM, Sinha R, Millikan RC, et al (2003). Heterocyclic amines, meat intake, and association with colon cancer in a population-based study. Am J Epidemiol, 157, 434-45.

Cross AJ, Ferrucci LM, Risch A, et al (2010). A large prospective study of meat consumption and colorectal cancer risk: an investigation of potential mechanisms underlying this association. Cancer Res, 70, 2406-14.

Gilsing AM, Berndt SI, Ruder EH, et al (2012). Meat-related mutagen exposure, xenobiotic metabolizing gene polymorphisms, and the risk of advanced colorectal adenoma and cancer. Carcinogenesis, 33, 1332-9.

Helmus DS, Thompson CL, Zelenskiy S, Tucker TC, Li L (2013). Red meat-derived heterocyclic amines increase the risk of colon cancer: a population-based case-control study. Nutr Cancer, 65, 1141-50.

Hodge JE (1953). Dehydrated foods, the chemistry of browning reactions in model systems. J Agric Food Chem, 1, 928-43.

IARC (1993). Some Naturally Occurring Substances: Food Items and Constituents, Heterocyclic Aromatic Amines, and Mycotoxins. In IARC Monographs on the Evaluation of Carcinogenic Risks to Humans, WHO-IARC (ed), Vol. 56. pp. 165-229. WHO-IARC: Lyon France.

IARC (2018). Red meat and processed meat / IARC Working Group on the Evaluation of Carcinogenic Risks to Humans. Vol. 114. IARC (International Agency for Research on Cancer): Lyon, France.

Joshi AD, Kim A, Lewinger JP, et al (2015). Meat intake, cooking methods, dietary carcinogens, and colorectal cancer risk: findings from the Colorectal Cancer Family Registry. Cancer Med, 4, 936-52.

Kobayashi M, Otani T, Iwasaki M, et al (2009). Association between dietary heterocyclic amine levels, genetic polymorphisms of NAT2, CYP1A1, and CYP1A2 and risk of colorectal cancer: a hospital-based case-control study in Japan. Scand J Gastroenterol, 44, 952-9.

Le Marchand L, Hankin JH, Pierce LM, et al (2002). Well-done red meat, metabolic phenotypes, and colorectal cancer in Hawaii. Mutat Res, 506-507, 205-14.

Le TN, Le XH, Pham VP, et al (2018). Reproducibility of a designed semi-quantitative food frequency questionnaire in general populations in the North Viet Nam. Southeast Asian J Sci, 6, 188-97.

Linh DT (2019). Study on PhIP (2-Amino-1-methyl-6phenylimidazo[4,5-b] pyridine) and MeIQx (2-Amino-3,8dimethylimidazo[4,5-f] quinoxaline) analysis method and their content in some processed food, Master Thesis. pp. 73. Hanoi University of Science and Technology: School of Biotechnology and Food Technology.

Maillard LC (1912). Action of amino acids on sugars. Formation of melanoidins in a methodical way. Compt Rend, 154, 66.

Miller PE, Lazarus P, Lesko SM, et al (2013). Meat-related 
compounds and colorectal cancer risk by anatomical subsite. Nutr Cancer, 65, 202-6.

Ministry of Health \& National Institute of Nutrition. (2010). National Nutritional Survey 2009-2010. Medical Publishing House: Hanoi city.

Ngoan LT, Thu NT, Lua NT, et al (2009). Cooking temperature, heat-generated carcinogens, and the risk of stomach and colorectal cancers. Asian Pac J Cancer Prev, 10, 83-6.

Nowell S, Coles B, Sinha R, et al (2002). Analysis of total meat intake and exposure to individual heterocyclic amines in a case-control study of colorectal cancer: contribution of metabolic variation to risk. Mutat Res, 506-507, 175-85.

NTP (2014). Report on Carcinogens, Thirteenth Edition: Heterocyclic Amines (Selected). In http://ntp.niehs.nih.gov/ pubhealth/roc/roc13/index.html. U.S. Department of Health and Human Services: National Toxicology Program

Ollberding NJ, Wilkens LR, Henderson BE, Kolonel LN, Le Marchand L (2012). Meat consumption, heterocyclic amines, and colorectal cancer risk: the Multiethnic Cohort Study. Int $J$ Cancer, 131, 1125-33.

Sinha R, Rothman N, Salmon CP, et al (1998). Heterocyclic amine content in beef cooked by different methods to varying degrees of doneness and gravy made from meat drippings. Food Chem Toxicol, 36, 279-87.

Sugimura T (1997). Overview of carcinogenic heterocyclic amines. Mutat Res, 376, 211-9.

Thuong NV (2018). Mortality from non-communicable diseases occurred during 2005-2014 in Nghe An province and evaluation of the interventions to improve the quality of death registration. In Dept. of Occupational Health, Ph.D. Thesis. Hanoi Medical University, pp 147.

Turesky RJ, Goodenough AK, Ni W, et al (2007). Identification of 2-amino-1,7-dimethylimidazo[4,5-g]quinoxaline: an abundant mutagenic heterocyclic aromatic amine formed in cooked beef. Chem Res Toxicol, 20, 520-30.

\section{c) (7) (5)}

This work is licensed under a Creative Commons AttributionNon Commercial 4.0 International License. 